\title{
Design of Joint Sparse Graph for OFDM (JSG-OFDM) system
}

\author{
Lei Wen, Student Member, IEEE, Razieh Razavi, Member, IEEE, Muhammad Ali Imran, Senior Member, IEEE, \\ Pei Xiao, Senior Member, IEEE
}

\begin{abstract}
Low density signature orthogonal frequency division multiplexing (LDS-OFDM) and low density parity-check (LDPC) codes are multiple access and forward error correction (FEC) techniques, respectively. Both of them can be expressed by a bipartite graph. In this paper, we construct a joint sparse graph combining the single graphs of LDS-OFDM and LDPC codes, namely joint sparse graph for OFDM (JSG-OFDM). Based on the graph model, a low complexity approach for joint multiuser detection and FEC decoding (JMUDD) is presented. The iterative structure of JSG-OFDM receiver is illustrated, and its extrinsic information transfer (EXIT) chart is researched. Furthermore, design guidelines for the joint sparse graph are derived through the EXIT chart analysis. By offline optimization of the joint sparse graph, numerical results show that the JSG-OFDM brings about 1.5 - $1.8 \mathrm{~dB}$ performance improvement at bit error rate (BER) of $10^{-5}$ over similar well-known systems such as grouporthogonal multi-carrier code division multiple access (GO-MCCDMA), LDS-OFDM and turbo structured LDS-OFDM.
\end{abstract}

Index Terms-Multi access communication, Joint sparse graph, Joint multiuser detection and FEC decoding, Code division multiple access, Multiuser channels.

\section{INTRODUCTION}

O Ver the last decades, multi-carrier transmission has been considered as a promising technique for broadband wireless communications. Multi-carrier code division multiple access (MC-CDMA) and orthogonal frequency division multiplexing (OFDM) are suitable approaches to coping with high data-rate services, and they have been adopted as core technologies in some mobile communication standards, such as the 3rd Generation Partnership Project Long Term Evolution (3GPP-LTE) [1] and the Worldwide Interoperability for Microwave Access (WiMAX) [2]. Conventional MC-CDMA is based on orthogonality between spreading sequences to avoid multiuser interference (MUI). In uplink transmission, the number of users or parallel data streams inevitably exceeds the available dimensions as the demand for the spectrum is increasing while the bandwidth is fixed, which results in an overloaded condition. In that case, it is impossible to obtain the orthogonality of received signatures in MC-CDMA, consequently the the system performance is limited by the MUI. In this paper, we will discuss the issues related to the overloaded systems.

\section{A. Literature Review}

Linear constellation precoding for OFDM with a specifically chosen subcarrier grouping can achieve the maximum multipath diversity and coding gains as with precoding over all the available subcarriers, where the decoding complexity is approximately exponential in the channel order [3]. The idea of linear constellation precoding is similar to a real orthogonal precoder that can maximize the channel cutoff rate or the minimum product distance. In addition, multiuser detection is an effective way to eliminate the MUI. Linear detectors have been well-studied in the literature because of their simplicity. The most common linear detectors are the minimum mean square error (MMSE) detector [4] [5] and the decorrelator [6]. The MMSE detector implements the linear mapping which minimises the mean square error between the actual data and the soft outputs of the detector, and it provides good performance with much lower complexity compared to the optimum detector. The decorrelator inverts the channel matrix, but by doing so also enhances the noise. The advantage of the decorrelator is that no knowledge of the receive power is necessary and its performance is independent of the power of interfering users so that the near-far problem is avoided. However, all these linear detectors fail to perform satisfactorily under overloaded conditions as the desired (as well as the interferers') signal subspace become rank-deficient.

As for the class of nonlinear detectors, e.g., successive interference cancellation (SIC) [7] and parallel interference cancelation (PIC) [8], their performance is highly dependent on the first estimate being fed to the interference cancelation detector. Probabilistic data association (PDA) [9] and multidomain detectors [10] perform multiuser detection iteratively and have been shown to achieve a performance that is close to the optimum detector. Again, their performance degrades rapidly under overloaded conditions. In [11], an iterative interference cancellation method has been developed for an overloaded Walsh-Hadamard-spread MC-CDMA system. Another technique to handle overloaded conditions is the grouporthogonal MC-CDMA (GO-MC-CDMA) [12] [13]. It partitions the available subcarriers into groups and distributes users among the groups, then each group behaves as an independent MC-CDMA system with a smaller number of users. Furthermore, a class of low density signature (LDS) for overloaded MC-CDMA (LDS-OFDM) has been developed [14]-[17]. It is a special case of MC-CDMA, where its signature can be modelled as a bipartite graph ${ }^{1}$. Message passing algorithm (MPA), which is very efficient for belief propagation on sparse

\footnotetext{
${ }^{1} \mathrm{~A}$ bipartite graph is a graph (nodes connected by edges) whose nodes may be separated into two types, and edges only connect two nodes of different types.
} 
graphs ${ }^{2}$, is applied to perform multiuser detection in LDSOFDM. It has been shown numerically and analytically that the GO-MC-CDMA and the LDS-OFDM can attain good performance in overloaded conditions. As a drawback of nonlinear detectors, the receiver complexity becomes higher than that of linear detectors. Thus, it is important to strike a balance between performance and complexity when designing receivers.

In terms of receiver techniques, multiuser detection and forward error correction (FEC) decoding are arranged in MCCDMA to combat the MUI and fading as well as channel noise. In [18], a receiver that performs detection and decoding i) jointly (optimal) is referred to as Type-A receiver, ii) independently is referred to as Type-B receiver, and iii) iteratively (between detection and decoding) is referred to as Type-C receiver. Separation of detection and decoding (Type$\mathrm{B}$ receiver) significantly reduces the receiver complexity, but its performance is suboptimal. Obviously, GO-MC-CDMA and LDS-OFDM adopt Type-B receivers. Turbo equalization that performs detection and decoding in an iterative manner (Type-C receiver) is known to achieve better performance than the Type-B receiver. Turbo structured LDS-OFDM has been proposed in [17], where the information is exchanged between the detector and the decoder in a turbo manner. The receiver of the turbo structured LDS-OFDM is classified as Type-C and can improve the system performance. But it cannot perform joint detection and decoding of the Type-A receiver, meanwhile, its computational complexity is several times higher than that of the conventional LDS-OFDM. In the literature, a Type-A receiver, which takes into account the knowledge of channels, the FEC decoding, the de-spreading and the de-interleaving, usually becomes infeasible in practical systems, as it amounts to essentially trying to fit all possible sequences of transmitted bits to the received data - a task of prohibitive complexity. Therefore, the goal in this research is to design receivers which achieve near-optimum performance of the Type-A receiver with affordable computational costs.

\section{B. Novelties of This Paper}

The main contributions of this paper are summarized as follows.

1) The signature of LDS-OFDM is represented as a bipartite graph. Low density parity-check (LDPC) code, which is known to achieve capacity over additive white Gaussian noise (AWGN) channel, can also be expressed by a bipartite graph. According to their graph model, we propose to construct a joint sparse graph which includes the low density signature of LDS-OFDM and the low density parity-check matrices of LDPC codes. We refer it as joint sparse graph for OFDM (JSGOFDM). Unlike any existing sparse graph that is only used in one specific field such as LDS-OFDM, LDPC code, low density generator matrix (LDGM) code, repeat-accumulate (RA) code, Luby transform (LT) code and Raptor code, our

\footnotetext{
${ }^{2}$ Dense graph is a graph in which the number of edges is close to the maximal number of edges, thus it is a almost fully connected graph. The opposite, a graph with only a few edges, is a sparse graph. The distinction between dense and sparse graphs is the number of edges.
}

proposed JSG-OFDM is based on a novel joint sparse graph which combines multiple access and FEC techniques. The idea behind the joint sparse graph is basically to change the interference pattern being seen by each user, and limit the amount of interference occured on each chip.

2) To the best of our knowledge, there does not exist a multiple access system for which detection and decoding are performed simultaneously on one sparse graph. Based on the MPA and the joint sparse graph, we design a low complexity Type-A receiver for the JSG-OFDM. Joint multiuser detection and FEC decoding (JMUDD), which performs detection and decoding at the same time on the entire sparse graph, is presented. There are significant differences between the JSGOFDM and the LDS-OFDM presented in [17]. Assuming a LDPC code is used for FEC, the LDS-OFDM receiver introduced in [17] is a Type-B receiver, i.e., detection and decoding are performed and optimized separately. The turbo structured LDS-OFDM receiver developed in [17] is a Type-C receiver whose detection and decoding are performed iteratively in a turbo style. However, our proposed receiver of JSG-OFDM is a Type-A receiver based on a joint sparse graph, where detection and decoding are performed jointly on the entire graph. There is no turbo structure in the JSG-OFDM, but the detection and decoding information can be exchanged, thus the JSG-OFDM is a novel scheme and different from existing systems such as LDS-OFDM and turbo structured LDS-OFDM presented in [17].

3) Analysis of a joint sparse graph is different from that of a single sparse graph. We depict the iterative structure for JSGOFDM receiver in details, and use the extrinsic information transfer (EXIT) chart to analyse the convergence behavior of the JMUDD on the joint sparse graph.

4) According to the EXIT chart analysis, two important factors which affect the performance of the joint sparse graph are investigated: degree distributions and short cycles. As a result, design guidelines for the joint sparse graph are derived. With offline optimization of the joint sparse graph, JSGOFDM outperforms state-of-the-art multiple access systems. In other words, all the optimizations (for a wide range of parameters) are carried out in advance at the design stage and the receiver can use the optimum graph directly.

\section{Structure and Notations of This Paper}

The rest of this paper is organized as follows. Section II presents the JSG-OFDM system model. In Section III, the JMUDD on the joint sparse graph is presented. The EXIT chart for JMUDD on the joint sparse graph is analysed in Section IV, and the construction guidelines for a joint sparse graph are given in Section V. In Section VI, the performance and the detection complexity of JSG-OFDM are compared with existing multiple access systems. Finally, conclusions are drawn in Section VII.

In this paper, we use the following notations. Variables and constants are represented in lower case and upper case, respectively. Vectors and matrices are denoted by lower case and upper case, respectively, both in bold case. Vectors are assumed to be column. Superscript $T$ represents transpose of 
a vector or a matrix. The symbol $\propto$ means "is proportional to". A summary of notations used in this paper is presented in TABLE I.

\section{JSG-OFDM SYSTEM MODEL}

Consider the uplink communications with $K$ users transmitting to the same base station where the base station and each user are equipped with a single antenna. The block diagram of JSG-OFDM system is shown in Fig.1. Let the processing gain to be $N$, and each user has a data vector consisting of $M$ data symbols. Let $J$ be the number of parity-check equations in LDPC code. We assume that perfect channel state information is available at the receiver.

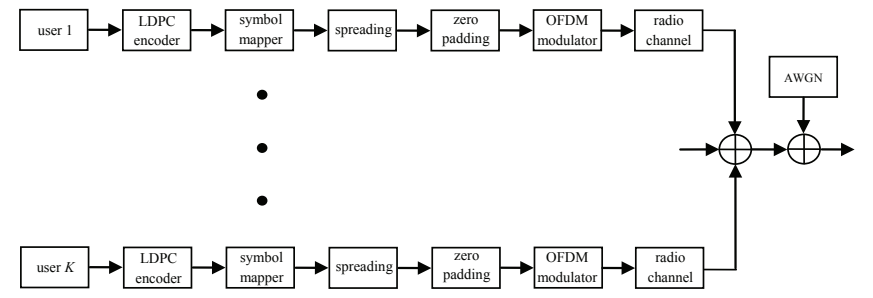

Block diagram for JSG-OFDM transmitter

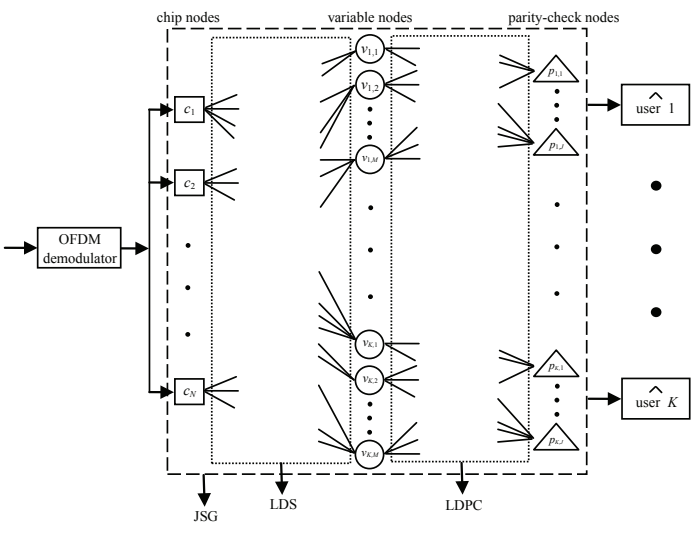

Block diagram for JSG-OFDM receiver

Fig. 1: JSG-OFDM system model

At the transmitter in Fig. 1, the functional blocks are similar to a MC-CDMA system. In conventional MC-CDMA, after FEC encoding and symbol mapping, data symbols are multiplied with a spreading signature (a random sequence of chips) and subsequently OFDM modulation is arranged to modulate the chips onto respective sub-carrier frequencies. The main difference in JSG-OFDM transmitter is that the spreading signature has low density by the use of zero padding, which means a large number of chips in the sequence are zeros. Due to the low density signature, each symbol is only spread over a limited number of chips. Each user's generated chip is transmitted over an orthogonal sub-carrier, and each subcarrier is only used by a limited number of symbols that may belong to different users. Each user, transmitting on given subcarriers, will experience interference from only a small number of other users' data symbols. In other words, the number of symbols that are superimposed on each chip is much less than the total number of symbols, $d_{c, l d s} \ll(K \times M)$, where $d_{c, l d s}$ is the number of symbols that are superimposed at one chip. Meanwhile, the number of chips that are spread by each symbol is much less than the total number of chips, $d_{v, l d s} \ll N$, where $d_{v, l d s}$ is the number of chips that are spread by one symbol. If $N$, the processing gain of spreading, is reduced, the system performance will degrade due to the loss of the spreading gain. $J$ is the number of redundant parity-check bits, thus the system performance will drop if $J$ is decreased. The ratio of $(K \times M)$ to $N$ is referred to as the system loading, if such ratio is decreased, the system performance can be improved.

As for the receiver in Fig. 1, there are three types of nodes: chip nodes $c_{n}(n \in[1, N])$, variable nodes $v_{k, m}(k \in$ $[1, K], m \in[1, M])$ and parity-check nodes $p_{k, j}(k \in$ $[1, K], j \in[1, J])$, representing the $n^{t h}$ chip, the $m^{t h}$ data symbol and the $j^{t h}$ parity-check equation of the $k^{t h}$ user, respectively. A single graph, as labelled with LDS in the receiver, represents the low density signature due to LDS-OFDM [16]. The other single graphs, as labelled with LDPC in the receiver, represent the low density parity-check matrices due to LDPC codes [19]. These two types of single graphs belong to the techniques of multiple access and FEC coding, respectively. In our proposal, variable nodes are used to connect the other two types of nodes (chip nodes and parity-check nodes) through low density edges. Therefore, the receiver becomes a joint sparse graph labelled with JSG in the figure. As such, the LDS structure and the LDPC codes are perfectly linked together. In the joint sparse graph, users' signals that are using the same chip will be superimposed, and the number of symbols that interfere with each other at one chip is much less than the total number of symbols, so the system can perform well in overloaded conditions. The joint sparse graph is arranged to process the chips from the received signals to reconstitute the transmitted data. It is noteworthy that applying MPA on the joint sparse graph performs not only detection, but also decoding at the same time. Furthermore, the receiver of JSGOFDM (Type-A receiver) is different from that of the turbo structured LDS-OFDM (Type-C receiver), as there is no outerinner turbo style iteration here. Hence, the JSG-OFDM is based on a joint sparse graph which combines multiple access and sparse graph coding techniques. To fit the stream format of practical communications, we can extend the data length, such that one frame contains multiple other than one OFDM symbol. In the next section, joint detection and decoding on such graph will be described.

\section{JMUDD FOR JSG-OFDM}

MPA, also known as belief propagation, calculates the marginal distribution for each node in graphical models. It is inefficient to apply the MPA for detection in conventional MC-CDMA system, as the spreading signature is a dense graph (almost fully connected graph), consequently leading to prohibitive computational complexity. Differing from conventional MC-CDMA, the joint sparse graph in JSG-OFDM has low density, thus iterative message passing becomes feasible and can attain near-optimum performance at low complexity. Based on the system model introduced in Section II, we 
TABLE I: Summary of key notations

\begin{tabular}{|c|c|c|c|}
\hline$K$ & Number of users & $r_{k, m}^{n}$ & Received signature gain at the $n^{t h}$ chip of the variable node $v_{k, m}$ \\
\hline$M$ & Data length of each user & $\mathbf{r}_{k, m}$ & Received spreading sequence for the data symbol $m$ of the $k^{t h}$ user \\
\hline$N$ & Number of chips & $y_{n}$ & Received signal corresponding to the $n^{t h}$ chip \\
\hline$J$ & Number of parity-check equations of LDPC codes & $z_{n}$ & AWGN \\
\hline$c_{n}$ & The $n^{t h}$ chip, also represents chip node & $\sigma_{A}^{2}$ & Variance of $z_{n}$ \\
\hline$v_{k, m}$ & $\begin{array}{l}\text { The } m^{t h} \text { data symbol of the } k^{t h} \text { user, also represents } \\
\text { variable node }\end{array}$ & $L_{v_{k, m} \rightarrow c_{n}}$ & LLR delivered from the variable node $v_{k, m}$ to the chip node $c_{n}$ \\
\hline$p_{k, j}$ & $\begin{array}{l}\text { The } j^{t h} \text { parity-check equation of the } k^{t h} \text { user, also repre- } \\
\text { sents parity-check node }\end{array}$ & $L_{v_{k, m}} \rightarrow p_{j}$ & $\begin{array}{l}\text { LLR delivered from the variable node } v_{k, m} \text { to the parity-check node } \\
p_{j}\end{array}$ \\
\hline$d_{c, l d s}$ & Number of symbols that are superimposed at one chip & $L_{c_{n} \rightarrow v_{k, m}}$ & LLR delivered from the chip node $c_{n}$ to the variable node $v_{k, m}$ \\
\hline$d_{v, l d s}$ & Number of chips that are spread by one symbol & $L_{p_{j} \rightarrow v_{k, m}}$ & $\begin{array}{l}\text { LLR delivered from the parity-check node } p_{j} \text { to the variable node } \\
v_{k, m}\end{array}$ \\
\hline$d_{v, l d p c}$ & $\begin{array}{l}\text { Number of parity-check nodes connected to one variable } \\
\text { node }\end{array}$ & $L_{v_{k, m}}$ & Final estimation of the variable node $v_{k, m}$ \\
\hline$d_{p, l d p c}$ & $\begin{array}{l}\text { Number of variable nodes connected to one parity-check } \\
\text { node }\end{array}$ & $\mathbf{v}$ & Transmitted vector \\
\hline $\mathbf{S}_{k}$ & Spreading matrix for the $k^{t h}$ user & $\mathbf{v}_{[n]}$ & $\begin{array}{l}\text { Vectors containing the symbols transmitted by every user that spread } \\
\text { its data on the } n^{t h} \text { chip }\end{array}$ \\
\hline $\mathbf{S}$ & Low density spreading signatures for OFDM & $\mathbf{r}_{[n]}$ & $\begin{array}{l}\text { Received signature vectors by every user that spread its data on the } \\
n^{t h} \text { chip }\end{array}$ \\
\hline $\mathbf{H}_{k}$ & Parity-check matrix for the $k^{t h}$ user & $\kappa_{n, k, m}$ & Normalization coefficient \\
\hline $\mathbf{H}$ & Low density parity-check matrices for LDPC codes & $\hat{v_{k, m}}$ & Estimated value of the variable node $v_{k, m}$ \\
\hline $\mathbf{T}$ & Transmit power gain & VNDD & Variable node detector-decoder \\
\hline $\mathbf{G}_{k}$ & Channel gain for the $k^{t h}$ user & CND & Chip nodes detector \\
\hline$\psi_{n}$ & Set of data symbols that interfere on chip $c_{n}$ & PND & Parity-check node decoder \\
\hline$\psi_{n} /(k, m)$ & $\begin{array}{l}\text { Set of data symbols (excluding } v_{k, m} \text { ) that interfere on chip } \\
c_{n}\end{array}$ & $I_{A, V N D D}$ & $\begin{array}{l}\text { Average mutual information between the bits on the VNDD edges and } \\
\text { the } a \text { priori LLR }\end{array}$ \\
\hline$\varepsilon_{k, m}$ & Set of chips that $v_{k, m}$ is spread on & $I_{E, V N D D}$ & $\begin{array}{l}\text { Average mutual information between the bits on the VNDD edges and } \\
\text { the extrinsic LLR }\end{array}$ \\
\hline$\varepsilon_{k, m} / n$ & Set of chips (excluding $c_{n}$ ) that $v_{k, m}$ is spread on & $I_{A, C N D \& P N D}$ & $\begin{array}{l}\text { Average mutual information between the bits on the CND\&PND edges } \\
\text { and the a priori LLR }\end{array}$ \\
\hline$\phi_{j}$ & Set of data symbols that connect to parity-check node $p_{k, j}$ & $I_{E, C N D \& P N D}$ & $\begin{array}{l}\text { Average mutual information between the bits on the CND\&PND edges } \\
\text { and the extrinsic LLR }\end{array}$ \\
\hline$\phi_{j} /(k, m)$ & $\begin{array}{l}\text { Set of data symbols (excluding } v_{k, m} \text { ) that connect to parity- } \\
\text { check node } p_{k, j}\end{array}$ & $D_{C N D}(x)$ & Degree distribution polynomials of chip nodes \\
\hline$\omega_{k, m}$ & Set of parity-check nodes that connect to $v_{k, m}$ & $D_{P N D}(x)$ & Degree distribution polynomials of parity-check nodes \\
\hline$\omega_{k, m} / j$ & $\begin{array}{l}\text { Set of parity-check nodes (excluding } p_{k, j} \text { ) that connect to } \\
v_{k, m}\end{array}$ & $D_{V N D D}(x)$ & Degree distribution polynomials of variable nodes \\
\hline
\end{tabular}

present JMUDD on the joint sparse graph. Note that our present system model is based on single antenna transmission where neither the transmitters nor receiver have multiple antennas, but the idea of the joint sparse graph can be extended to multiple-input and multiple-output (MIMO) systems, which needs more careful design of the space diversity.

Spreading signature and parity-check matrix for the $k^{\text {th }}$ user are $\mathbf{S}_{k}=\left[\mathbf{s}_{k, 1}, \ldots, \mathbf{s}_{k, M}\right] \in \mathbb{C}^{N \times M}$ and $\mathbf{H}_{k}=$ $\left[\mathbf{h}_{k, 1}, \ldots, \mathbf{h}_{k, J}\right] \in \mathbb{C}^{J \times M}$, respectively, where $\mathbb{C}$ denotes the complex field. Let $\mathbf{S}=\left[\mathbf{S}_{1}, \ldots, \mathbf{S}_{K}\right] \in \mathbb{C}^{N \times M \times K}$ and $\mathbf{H}=\left[\mathbf{H}_{1}, \ldots, \mathbf{H}_{K}\right] \in \mathbb{C}^{J \times M \times K}$ be the low density spreading signatures for OFDM and the low density parity-check matrices for LDPC codes, respectively. We also define $\mathbf{T}=$ $\operatorname{diag}\left(\mathbf{T}_{1}, \ldots, \mathbf{T}_{K}\right)$ as the transmit power gain of users and $\mathbf{G}_{k}=\operatorname{diag}\left(\mathbf{g}_{k, 1}, \ldots, \mathbf{g}_{k, N}\right)$ as the corresponding channel gain for the $k^{t h}$ user. Moreover, $\psi_{n}=\left\{(k, m): \mathbf{s}_{k, m}^{n} \neq 0\right\}$ and $\varepsilon_{k, m}=\left\{n: \mathbf{s}_{k, m}^{n} \neq 0\right\}$ are the set of data symbols (which may belong to different users) that interfere on chip $c_{n}$ and the set of chips that $v_{k, m}$ is spread on, respectively; $\phi_{j}=\left\{(k, m): \mathbf{h}_{k, m}^{j} \neq 0\right\}$ and $\omega_{k, m}=\left\{j: \mathbf{h}_{k, m}^{j} \neq 0\right\}$ are the set of data symbols that connect to parity-check node $p_{k, j}$ and the set of parity-check nodes that connect to $v_{k, m}$, respectively.
In JSG-OFDM, each user's chip will be transmitted over an orthogonal sub-carrier. Therefore, the received spreading sequence for the data symbol $m$ of the $k^{t h}$ user can be represented by $\mathbf{r}_{k, m}=\mathbf{T}_{k} \mathbf{G}_{k} \mathbf{s}_{k, m}$. In particular, the received signature gain at the $n^{t h}$ chip of the variable node $v_{k, m}$ is $\mathbf{r}_{k, m}^{n}=\mathbf{T}_{k} \mathbf{g}_{k, n} s_{k, m}^{n}$. For the uplink MC-CDMA, the received signal corresponding to the $n^{\text {th }}$ chip (sub-carrier) is written as

$$
y_{n}=\sum_{k=1}^{K} \sum_{m=1}^{M} \mathbf{r}_{k, m}^{n} v_{k, m}+z_{n}
$$

where $z_{n}$ is the AWGN with variance $\sigma_{A}^{2}$ and mean zero. Considering that in JSG-OFDM, the signature has a limited number of non-zero positions, we can express the received signal at the $n^{\text {th }}$ chip (sub-carrier) as

$$
y_{n}=\sum_{(k, m) \in \psi_{n}} \mathbf{r}_{k, m}^{n} v_{k, m}+z_{n}
$$

Let $L_{v_{k, m} \rightarrow c_{n}}$ and $L_{v_{k, m} \rightarrow p_{k, j}}$ be the log-likelihood ratio (LLR) delivered from the variable node $v_{k, m}$ to the chip node $c_{n}$ and the parity-check node $p_{k, j}$, respectively. The LLR delivered from the chip node $c_{n}$ and the parity-check node $p_{k, j}$ to the variable node $v_{k, m}$ are given by $L_{c_{n} \rightarrow v_{k, m}}$ and 
$L_{p_{k, j} \rightarrow v_{k, m}}$, respectively. $L_{v_{k, m}}$ is the final estimation of the variable node $v_{k, m}$. In a typical run, each message will be updated iteratively from the previous values of the neighboring LLR. The JMUDD can be presented as messages updating between different types of nodes via edges, which is explained as follows.

\section{A. Initialization}

Assuming there is no a priori probability available, initial LLR are set to zeros.

$$
L_{v_{k, m} \rightarrow c_{n}}=0, L_{v_{k, m} \rightarrow p_{k, j}}=0, \forall k, \forall m, \forall n, \forall j
$$

\section{B. Updating of Chip Nodes and Parity-Check Nodes}

LLR of the chip nodes and the parity-check nodes are calculated at the same time. For the chip nodes,

$$
L_{c_{n} \rightarrow v_{k, m}} \propto f\left(v_{k, m} \mid y_{n}, L_{v_{k^{\prime}, m^{\prime}} \rightarrow c_{n}},\left(k^{\prime}, m^{\prime}\right) \in \psi_{n} \backslash(k, m)\right)
$$

where $\psi_{n} \backslash(k, m)$ is the set of data symbols (excluding $v_{k, m}$ ) that interfere on the chip $c_{n}$. In order to approximate the maximum a posteriori (MAP) probability detector, the right hand side of (4) represents marginalization function, which is based on (2), and can be written as

$$
\begin{aligned}
& f\left(v_{k, m} \mid y_{n}, L_{v_{k^{\prime}, m^{\prime}} \rightarrow c_{n}},\left(k^{\prime}, m^{\prime}\right) \in \psi_{n} \backslash(k, m)\right) \\
& =\log \left(\sum p\left(y_{n} \mid \mathbf{v}\right) p_{n}\left(\mathbf{v} \mid v_{k, m}\right)\right) \\
& =\log \left(\sum p\left(y_{n} \mid \mathbf{v}\right) \prod_{\left(k^{\prime}, m^{\prime}\right) \in \psi_{n} \backslash(k, m)} p_{n}\left(v_{k^{\prime}, m^{\prime}}\right)\right)
\end{aligned}
$$

where $\mathbf{v}$ is the transmitted vector, the conditional probability density function $p\left(y_{n} \mid \mathbf{v}\right)$ and a priori probability $p_{n}\left(v_{k^{\prime}, m^{\prime}}\right)$ are given as

$$
\begin{gathered}
p\left(y_{n} \mid \mathbf{v}\right) \propto \exp \left(-\frac{1}{2 \sigma_{A}^{2}}\left\|y_{n}-\mathbf{r}_{[n]}^{T} \mathbf{v}_{[n]}\right\|^{2}\right) \\
p_{n}\left(v_{k^{\prime}, m^{\prime}}\right)=\exp \left(L_{v_{k^{\prime}, m^{\prime} \rightarrow c_{n}}}\right)
\end{gathered}
$$

where $\mathbf{v}_{[n]}$ and $\mathbf{r}_{[n]}$ denote the vector containing the symbols transmitted by every user that spread its data on the $n^{\text {th }}$ chip and their corresponding effective received signature values, respectively. As can be seen from (5), based on the received chip $y_{n}$ and a priori input information $p_{n}\left(v_{k^{\prime}, m^{\prime}}\right)$, extrinsic values are calculated for all the constituent bits involved in (2). Substituting (6) and (7) into (5), the message update becomes

$$
\begin{aligned}
& L_{c_{n} \rightarrow v_{k, m}}=\kappa_{n, k, m} \max _{\mathbf{v}_{[n]}}{ }^{*}\left(\sum_{\left(k^{\prime}, m^{\prime}\right) \in \psi_{n} \backslash(k, m)} L_{v_{k^{\prime}, m^{\prime}} \rightarrow c_{n}}\right. \\
& \left.-\frac{1}{2 \sigma_{A}^{2}}\left\|y_{n}-\mathbf{r}_{[n]}^{T} \mathbf{v}_{[n]}\right\|^{2}\right)
\end{aligned}
$$

where $\kappa_{n, k, m}$ denotes the normalization coefficient and

$$
\max ^{*}(a, b) \triangleq \log \left(e^{a}+e^{b}\right)=\max (a, b)+\log \left(1+e^{-|a-b|}\right)
$$

The LLR of the parity-check nodes is updated as

$$
L_{p_{k, j} \rightarrow v_{k, m}}=\alpha^{-1}\left(\sum_{\left(k^{\prime}, m^{\prime}\right) \in \phi_{j} \backslash(k, m)} \alpha\left(L_{v_{k^{\prime}, m^{\prime}} \rightarrow p_{k, j}}\right)\right)
$$

where $\phi_{j} \backslash(k, m)$ is the set of data symbols (excluding $v_{k, m}$ ) that connect to the parity-check node $p_{k, j}$, and

$$
\alpha(x)=\operatorname{sign}(x) \times\left(-\log \tan \frac{|x|}{2}\right)
$$

where $\operatorname{sign}(x)$ represents the sign of $x$, and the inverse of $\alpha(x)$ is

$$
\alpha^{-1}(x)=(-1)^{\operatorname{sign}(x)} \times\left(-\log \tan \frac{|x|}{2}\right)
$$

\section{Updating of Variable Nodes}

In single graph case, variable nodes only gather information from one type of nodes (chip nodes or parity-check nodes) [16] [19]. However, in the joint sparse graph, the updating of $L_{v_{k, m} \rightarrow c_{n}}$ not only receives chip nodes information, but also ultilizes the information that comes from parity-check nodes.

$$
L_{v_{k, m} \rightarrow c_{n}}=\sum_{n^{\prime} \in \varepsilon_{k, m} \backslash n} L_{c_{n^{\prime}} \rightarrow v_{k, m}}+\sum_{j \in \omega_{k, m}} L_{p_{k, j} \rightarrow v_{k, m}}
$$

where $\varepsilon_{k, m} \backslash n$ is the set of chips (excluding $c_{n}$ ) that $v_{k, m}$ is spread on.

Similarly, calculation of $L_{v_{k, m} \rightarrow p_{k, j}}$ also involves the information from both sides, i.e.

$$
L_{v_{k, m} \rightarrow p_{k, j}}=\sum_{j^{\prime} \in \omega_{k, m} \backslash j} L_{p_{k, j^{\prime}} \rightarrow v_{k, m}}+\sum_{n \in \varepsilon_{k, m}} L_{c_{n} \rightarrow v_{k, m}}
$$

where $\omega_{k, m} \backslash j$ is the set of parity-check nodes (excluding $p_{k, j}$ ) that connect to the variable node $v_{k, m}$.

\section{Estimation and Syndrome Computing}

In the single graph case of LDS-OFDM, a posteriori probability of the transmitted symbol can only be calculated after a fixed number of iterations, as there is no criterion to determine whether the iterative message has converged [16]. Fortunately, in the joint sparse graph, parity-check nodes are available, thus it is possible to terminate the JMUDD process by syndrome computing. a posteriori probability of the transmitted symbo $v_{k, m}$ is calculated as

$$
L_{v_{k, m}}=\sum_{n \in \varepsilon_{k, m}} L_{c_{n} \rightarrow v_{k, m}}+\sum_{j \in \omega_{k, m}} L_{p_{k, j} \rightarrow v_{k, m}}
$$

The estimated value of the variable node $v_{k, m}$ is obtained by making a hard decision,

$$
\hat{v_{k, m}}=\arg \max _{v_{k, m}} L_{v_{k, m}}
$$

If the result of syndrome computing for each user equals to zero, or the maximum iteration number is reached, the process is terminated. Otherwise, the iteration goes on. 
One of the advantages of the iterative receiver for JSGOFDM is its ability to support high loads while maintaining satisfactory performance and affordable complexity. In the next section, we carry out theoretical analysis of the joint sparse graph using EXIT chart.

\section{EXIT CHART ANALYSIS OF JSG-OFDM}

EXIT chart is a useful tool to analyse the transfer of information between the soft-input soft-output (SISO) constituents, and it provides an approximate visualization of the process of belief propagation. However, EXIT chart has not been applied to the joint sparse graph. In this section, we shall explain how EXIT charts can be utilized to analyse the convergence behavior of JMUDD in JSG-OFDM.

\section{A. Iterative Structure of the Joint Sparse Graph}

Before applying EXIT charts to the JSG-OFDM, we depict the iterative structure of the joint sparse graph. According to the JMUDD presented in Section III, variable nodes calculate the extrinsic messages to chip nodes using a priori information which they receive from other connected chip nodes and parity-check nodes. Meanwhile, based on the received a priori information, variable nodes calculate the extrinsic messages to parity-check nodes. The same rule applies to the extrinsic messages that the chip nodes and parity-check nodes send to variable nodes. To evaluate the transformation of extrinsic information in the joint sparse graph, the sets of chip nodes, variable nodes and parity-check nodes are referred to as chip nodes detector (CND), variable node detector-decoder (VNDD) and parity-check node decoder (PND), respectively. Fig. 2 shows the structure of the iterative detector and decoder in JSG-OFDM. As depicted in the figure, the extrinsic LLR that has been passed on are considered as a priori information by the other detector or decoder. The edge interleavers connect the different type of nodes, each of which represents a sparse signature or matrix. It is worth noting that such iterative structure is more complicated than any previous single graph which only has the left part labelled as LDS [17] or the right part labelled as LDPC [20] in this figure.

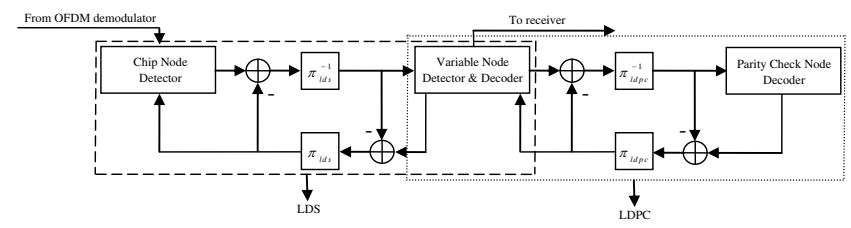

Fig. 2: Iterative structure of JMUDD in JSG-OFDM

Additionally, Fig. 3 shows a folded view of the joint sparse graph. Based on the JMUDD presented in Section III, chip nodes and parity check nodes update their messages at the same time, then variable nodes calculate the LLR delivered to chip nodes and parity check nodes simultaneously. Therefore, it is reasonable to place chip nodes (rectangles) and paritycheck nodes (triangles) on one side, while to draw variable nodes (circles) on the other side. The chip nodes are used to be spread on for the variable nodes through low density edges, which are represented by bold lines. The parity-check nodes, belonging to different users, are connected to corresponding groups of variable nodes through sparse edges, which are represented by independent groups of dash lines. Multiple access, FEC coding and the combination of several single sparse graphs, are clearly depicted in the figure. This provides a basis for the following analysis.

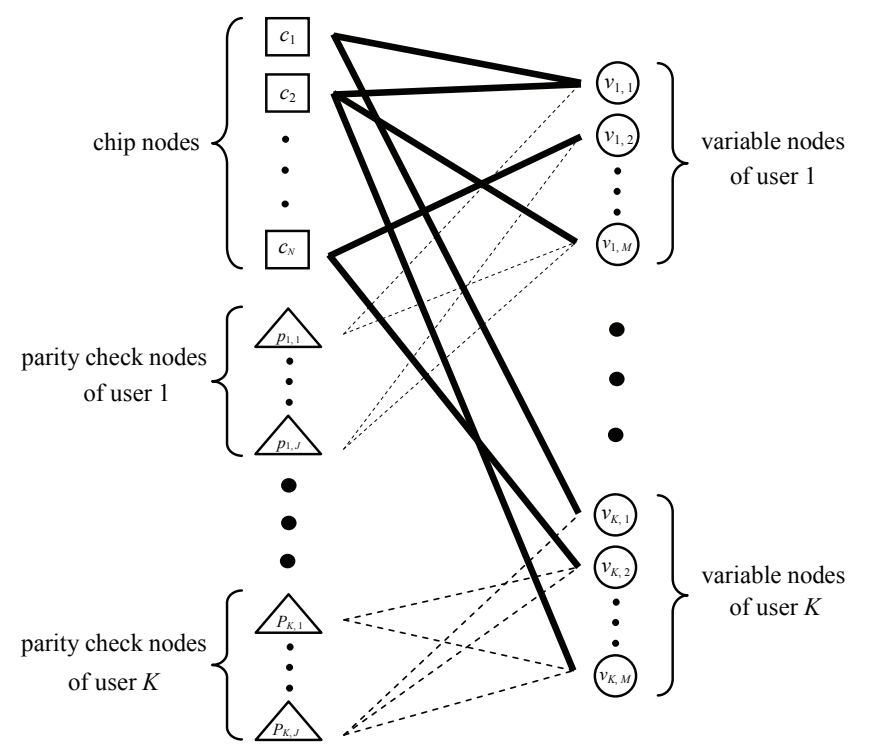

Fig. 3: Folded view of the joint sparse graph

\section{B. EXIT Chart Analysis Over AWGN Channel}

1) EXIT Curve for VNDD: In this paper, $I_{A, V N D D}$ refers to the average mutual information between the bits on the VNDD edges and the a priori LLR, $I_{E, V N D D}$ is the average mutual information between the bits on the VNDD edges and the extrinsic LLR. In order to compute an EXIT curve for variable nodes, $L_{c_{n} \rightarrow v_{k, m}}$ and $L_{p_{k, j} \rightarrow v_{k, m}}$ are modelled as the soft output of an AWGN channel when the inputs are interleaved bit transmitted using binary phase shift keying (BPSK). Then the mutual information between the variable node's extrinsic messages and actual values of symbols on the edges is calculated. A priori LLR can be calculated by

$$
A=\mu_{A} x+z_{n}
$$

where $z_{n}$ is an independent Gaussian random variable with variance $\sigma_{A}^{2}$ and mean zero; $x \in \pm 1$ is the original bit on the graph edge. Furthermore, we have

$$
\mu_{A}=\frac{\sigma_{A}^{2}}{2}
$$

The mutual information $I_{A, V N D D}=I(X ; A)$ can be calculated by

$$
\begin{aligned}
I_{A, V N D D}= & \frac{1}{2} \sum_{x=-1,1} \int_{-\infty}^{+\infty} p_{A}(\beta \mid X=x) \log _{2} \\
& \frac{2 p_{A}(\beta \mid X=x)}{p_{A}(\beta \mid X=-1)+p_{A}(\beta \mid X=1)} d \beta
\end{aligned}
$$


Since the conditional probability density function $p_{A}(\beta \mid X=x)$ depends on LLR of $A$, we can write

$$
\begin{aligned}
I_{A, V N D D}\left(\sigma_{A}\right)= & 1-\int_{-\infty}^{+\infty} \frac{e^{-\left(\left(\beta-\sigma_{A}^{2} / 2\right)^{2} / 2 \sigma_{A}^{2}\right)}}{\sqrt{2 \pi} \sigma_{A}} \log _{2} \\
& \left(1+e^{-\beta}\right) d \beta
\end{aligned}
$$

For abbreviation we define

$$
B(\sigma):=I_{A, V N D D}\left(\sigma_{A}=\sigma\right)
$$

with

$$
\begin{aligned}
& \lim _{\sigma \rightarrow 0} B(\sigma)=0 \\
& \lim _{\sigma \rightarrow \infty} B(\sigma)=1
\end{aligned}
$$

where $\sigma \geq 0$. Considering (13) and (14) together with the fact that the sum of two normally distributed random variables is also normally distributed with the mean and variance equal to the sum of theirs, the EXIT function of a variable node can be expressed as

$$
\begin{aligned}
& I_{E, V N D D}\left(I_{A, V N D D}, d_{v, l d s}, d_{v, l d p c}\right)= \\
& B\left(\sqrt{\left(d_{v, l d s}+d_{v, l d p c}-1\right)\left(B^{-1}\left(I_{A, V N D D}\right)\right)^{2}}\right)
\end{aligned}
$$

where $d_{v, l d s}$ is the effective spreading factor and $d_{v, l d p c}$ is the number of parity-check nodes connected to one variable node. Therefore, unlike single sparse graph where only one type of node is considered [16] [20], both LDS and LDPC nodes affect the VNDD performance.

2) EXIT Curve for CND\&PND: Let $I_{A, C N D \& P N D}$ refers to the average mutual information between the bits on the CND\&PND edges and the a priori LLR, $I_{E, C N D \& P N D}$ is the average mutual information between the bits on the CND\&PND edges and the extrinsic LLR. A chip node has incoming messages from the connected variable nodes and the OFDM demodulator, whereas a parity-check node only has messages coming from neighbored variable nodes. The output LLR of chip nodes and parity-check nodes are calculated by (8) and (10), respectively. We model $L_{v_{k, m} \rightarrow c_{n}}$ and $L_{v_{k, m} \rightarrow p_{k, j}}$ as the output of an AWGN channel that the input is the corresponding transmitted bit using BPSK, and then calculate the mutual information of the output with regards to the actual value on the edges. Due to the complexity of the calculation in chip nodes and parity-check nodes, their EXIT curves are computed by simulations over AWGN channel. The probability density function for extrinsic information is determined by Monte Carlo simulation with histogram measurements, the mutual information between the extrinsic information and the bits on the joint graph edges, is subsequently calculated.

3) Analysis: System paramters are listed in TABLE II for the following EXIT chart analysis. The effective spreading factor is set to 3 in order to achieve a good trade-off between frequency diversity and complexity [17]. Fig. 4 illustrates the EXIT charts over AWGN channel at $E_{b} / N_{0}=9 \mathrm{~dB}$. For comparison, we also plot curves of LDS-OFDM system, where there are only two sets of nodes in the single graph of LDSOFDM: chip node detector (CND) and variable node detector (VND). According to Fig. 4, we can concluded:

TABLE II: System parameters

\begin{tabular}{|c|c|}
\hline Number of users & 6 \\
\hline Number of chip nodes & 120 \\
\hline Number of variable nodes & 240 \\
\hline Number of parity-check nodes & 120 \\
\hline FFT size & 128 \\
\hline Sub-channel bandwidth & $15 \mathrm{KHz}$ \\
\hline System loading & $200 \%$ \\
\hline Number of variable nodes connected to each chip node & $d_{c, l d s}=6$ \\
\hline Effective spreading factor & $d_{v, l d s}=3$ \\
\hline Number of parity-check nodes connected to each variable node & $d_{v, l d p c}=3$ \\
\hline Number of variable nodes connected to each parity-check node & $d_{p, l d p c}=6$ \\
\hline Modulation & BPSK \\
\hline
\end{tabular}

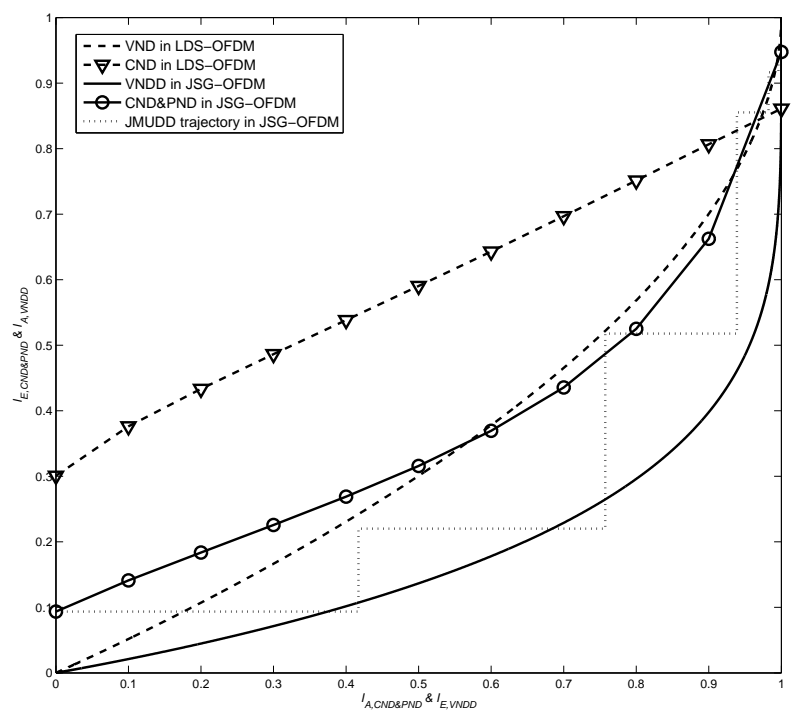

Fig. 4: EXIT chart over AWGN Channel at $E_{b} / N_{0}=9 \mathrm{~dB}$

1) The curve of VND in LDS-OFDM is higher than that of VNDD in JSG-OFDM. This is because the edge numbers connecting to variable nodes are different between single graph case of LDS-OFDM and joint graph case of JSG-OFDM, which has seen illustrated in Fig. 2 and Fig. 3. As a result, according to (24), these two curves are different.

2) The curve of CND in LDS-OFDM is higher than that of CND\&PND in JSG-OFDM when $I_{A, C N D \& P N D} \leq 0.95$. This follows from the fact that the CND could receive information from both OFDM demodulator and neighbored variable nodes, but the PND only uses the information from the connected variable nodes (shown in Fig. 1). Hence, the PND pulls down the average extrinsic information of CND\&PND in JSGOFDM at the first few iterations.

3) The intersection point of VNDD and CND\&PND in JSG-OFDM, is higher than that of VND and CND in LDSOFDM. This phenomenon implies that the JSG-OFDM has 
better ability to eliminate the MUI than LDS-OFDM.

4) The simulation trajectory of JMUDD in JSG-OFDM is also plotted in Fig. 4, which is marked by the dotted line. We can see that the iterative process starts with $I_{A, C N D \& P N D}=0$ since no prior information is available for the CND\&PND in the beginning. In the following steps, the output LLR is exchanged between the two solid curves. The trajectory closely follows the transfer curves of the components in JSG-OFDM, which indicates that the EXIT charts analysis is valid for the joint sparse graph. The minor discrepancy is due to the finite size of edge interleaver in the joint sparse graph.

\section{EXIT Chart Analysis Over Multipath Fading Channels}

As OFDM is used to combat the negative effect of multipath, we analyse the EXIT chart over multipath fading channels in the sequel. Note that the EXIT chart technique is not limited to the AWGN channel, it can also be applied to multipath fading channels when perfect channel state information is available at the receiver [21]. An EXIT chart assumes that the probability density function of the exchanged messages approaches a Gaussian-like distributions with increasing number of iterations. Consequently, it can be applied to multipath fading channels as long as the trajectory follows the curves of the receiver components. Similar to the analysis for the AWGN channel, the EXIT function of a variable node is the same as (24), which means the fading does not have any effect on the performance of VNDD, however its effect will be on the CND\&PND because it calculates its messages based on the received signal from the fading channel. Due to the complexity of calculation in (8) and (10), their EXIT curves are drawn by simulations. Fig. 5 illustrates the EXIT charts for LDS-OFDM and JSG-OFDM over ITU Pedestrian Channel $\mathrm{B}$, a typical multipath fading channel model, at $E_{b} / N_{0}=13$ $\mathrm{dB}$. The number of channel taps equal to six. As shown in the figure, JSG-OFDM has a higher intersection point than LDS-OFDM. Fig. 5 also shows the simulation trajectory of JMUDD for JSG-OFDM, which is marked by the dotted line. Thus the EIXT chart analysis is verified and visualized by the simulated trajectory, and the actual trajectory of JMUDD is well predicted at various iterations with only a marginal difference. This validates the EXIT chart analysis for multipath fading channels, and it will be further confirmed in Section VI.

\section{EXIT Chart BAsed Design of JoInt Sparse Graph}

According to graph theory, there are different parameters affecting the performance of a sparse graph. Two important factors are degree distributions of nodes and short cycles [22]. In this section, EXIT charts are used for the optimization of the joint sparse graph.

\section{A. Degree Distribution}

Degree of a node is the number of edges the node connected to other kinds of nodes, while degree distribution is the probability distribution of these degrees in a graph model. For the joint sparse graph, let $D_{C N D}(x), D_{P N D}(x)$ and $D_{V N D D}(x)$ denote the degree distribution polynomials of chip

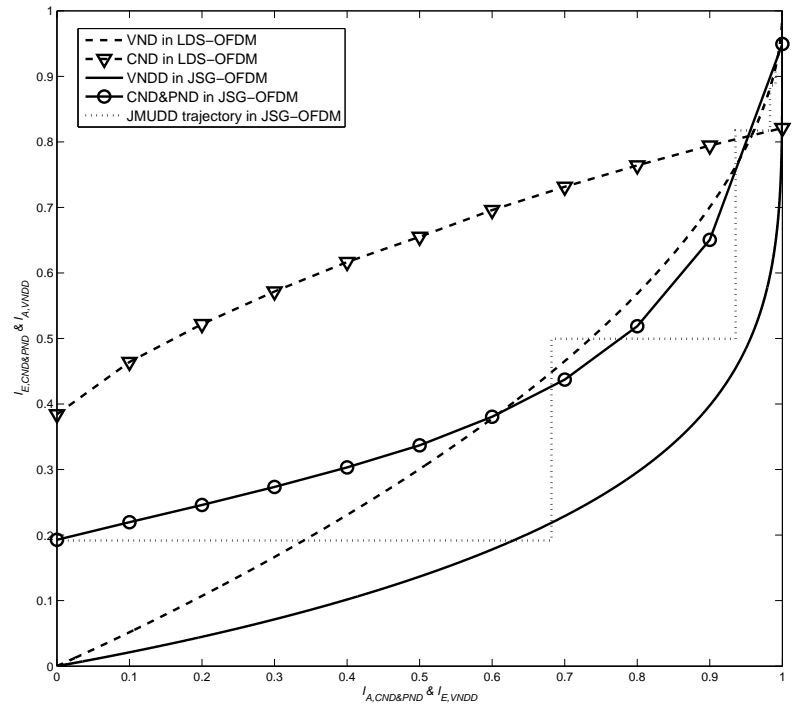

Fig. 5: EXIT chart over ITU Pedestrian Channel B at $E_{b} / N_{0}$ $=13 \mathrm{~dB}$

nodes, parity-check nodes and variable nodes, respectively. They are defined as

$$
\begin{gathered}
D_{C N D}(x)=\sum_{d=1}^{d_{c, l d s}} P_{C N D} x^{d-1} \\
D_{P N D}(x)=\sum_{d=1}^{d_{p, l d p c}} P_{P N D} x^{d-1} \\
D_{V N D D}(x)=\sum_{d=1}^{d_{v, l d s}+d_{v, l d p c}} P_{V N D D} x^{d-1}
\end{gathered}
$$

where $P_{C N D}, P_{P N D}$ and $P_{V N D D}$ are ractions of edges related to corresponding nodes. As 3 is suitable for the effective spreading factor, we fix $d_{v, l d s}=3$. However, other degree distributions can be optimized.

In information theory, the mutual information is a measure of the variables' mutual dependence. Ideally, in order to the exchange extrinsic information between the components to a convergence point such that an arbitrarily low bit error rate (BER) can be achieved, the EXIT curves should not intersect before reaching the $\left(I_{A}, I_{E}\right)=(1,1)$ point [21] [23] [24]. This implies that given $I_{A}=1$, we have $I_{E}=1$ and provided that this condition is satisfied, a so-called open-convergence tunnel appears in the EXIT chart. If however, the two curves intersect at a point lower than the $(1,1)$ point, it forms a semi-convergence tunnel, ant it will yield a higher BER than the scheme with an intersection at the $(1,1)$ point. In order to investigate how the position of the intersection point in the EXIT chart affects the BER performance, Fig. 6 shows the achievable BER as a function of the average mutual information $I_{A, V N D D}$ for the joint sparse graph presented in TABLE II over ITU Pedestrian Channel B. This figure gives an indication of the minimum required $I_{A, V N D D}$ in order to 
achieve a target BER. For example, to achieve the BER of $10^{-3} / 10^{-4} / 10^{-5}$, the point of intersection should be at least at $I_{A, V N D D}=0.86 / 0.93 / 0.96$. Hence, to reach the maximum dependence between detected symbols and their real values, the goal of an iterative system is to approach the $(1,1)$ point in the EXIT chart.

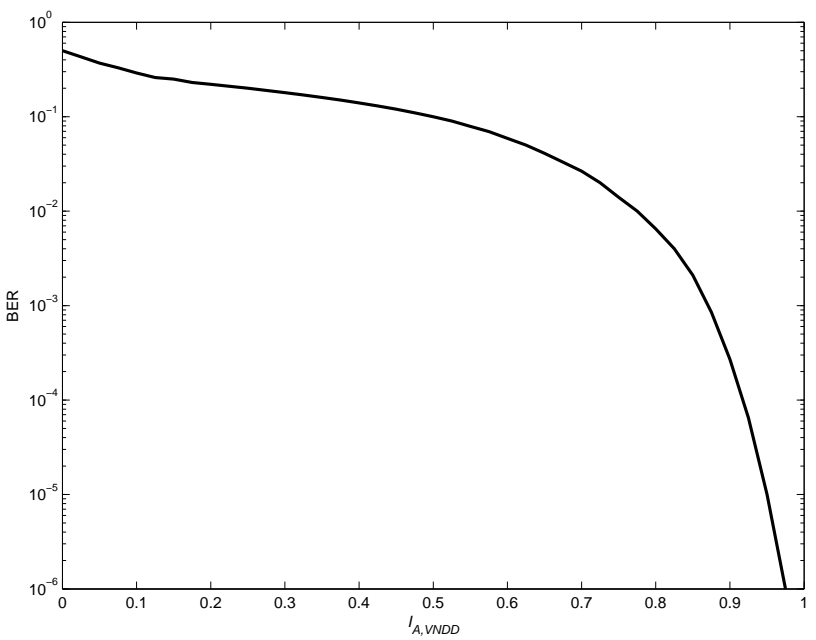

Fig. 6: BER versus $I_{A, V N D D}$ for the joint sparse graph

The joint sparse graph presented in TABLE II is a regular graph $^{3}$. Although its intersection point is higher than that of LDS-OFDM, which is shown in Fig. 4 and Fig. 5, the average level of CND\&PND in JSG-OFDM is much lower than that of CND in LDS-OFDM. Our approach is based on invoking EXIT chart analysis for optimizing the shape of the EXIT tunnel in order to achieve the $(1,1)$ point. As can be seen from (8), the information coming from the OFDM demodulator serves as input source of the joint sparse graph, and it is fed into CND in each iteration of the JMUDD. According to Section IV, the CND could receive information from both OFDM demodulator and neighbouring variable nodes, but the PND only uses the information from the connected variable nodes excluding direct channel knowledge, thus the PND pulls down the average extrinsic information of CND\&PND in JSGOFDM at the first few iterations. As mentioned previously, the mutual information determines the dependence between detected symbols and their exact values, thus the EXIT curve level has to be considered. If the proportion of the edges related to PND is reduced properly, the curve of CND\&PND can be lifted up, and the adjusted curves of the EXIT chart enable us to create a near-capacity scheme. As a further benefit, we are able to shift the EXIT functions close to the $(1,1)$ point in order to obtain a lower BER. Based on above analysis, several schemes of degree distribution are shown in TABLE III, where $\operatorname{Deg}_{a}$ is the case of a regular joint sparse graph presented in TABLE II, other schemes are for irregular joint sparse graphs. We can see that compared to $D e g_{a}, D e g_{c}$ and $D e g_{d}$ slightly decrease the degree of PND,

\footnotetext{
${ }^{3}$ If the degrees are constant values, the graph is a regular graph. Otherwise it is an irregular graph.
}

then the polynomials of VNDD are altered accordingly. On the contrary, $\mathrm{Deg}_{b}$ increases the density of edges of PND.

TABLE III: Degree distributions

\begin{tabular}{|c|c|c|c|}
\hline Scheme & $D_{C N D}(x)$ & $D_{P N D}(x)$ & $D_{V N D D}(x)$ \\
\hline$D e g_{a}$ & $x^{5}$ & $x^{5}$ & $x^{5}$ \\
\hline$D e g_{b}$ & $0.15 x^{4}+0.7 x^{5}+0.15 x^{6}$ & $0.09 x^{5}+0.91 x^{6}$ & $0.5 x^{5}+0.5 x^{6}$ \\
\hline$D e g_{c}$ & $0.05 x^{4}+0.9 x^{5}+0.05 x^{6}$ & $0.4 x^{3}+0.6 x^{4}$ & $0.7 x^{4}+0.3 x^{5}$ \\
\hline$D e g_{d}$ & $0.03 x^{4}+0.94 x^{5}+0.03 x^{6}$ & $0.8 x^{3}+0.2 x^{4}$ & $0.9 x^{4}+0.1 x^{5}$ \\
\hline
\end{tabular}

Fig. 7 shows EXIT charts of different degree distribution over ITU Pedestrian Channel B at $E_{b} / N_{0}=13 \mathrm{~dB}$. For $D e g_{c}$ and $D e g_{d}$, the proportion of the edges related to PND is reduced, consequently, as seen in Fig. 7, the average mutual information between the bits and the extrinsic values of CND\&PND are both increased. More importantly, their intersection point becomes higher and closer to the $(1,1)$ point than that of $D e g_{a}$, which means better performance can be achieved. By contrast, $D e g_{b}$ increases the density of edges of PND, but its average mutual information and intersection point are both dropped. Thus degree distribution is a key factor to the performance of JSG-OFDM. Although the curves of $D e g_{c}$ and $D e g_{d}$ almost overlap, $D e g_{d}$ is a more suitable choice since it has the lowest density among these schemes.

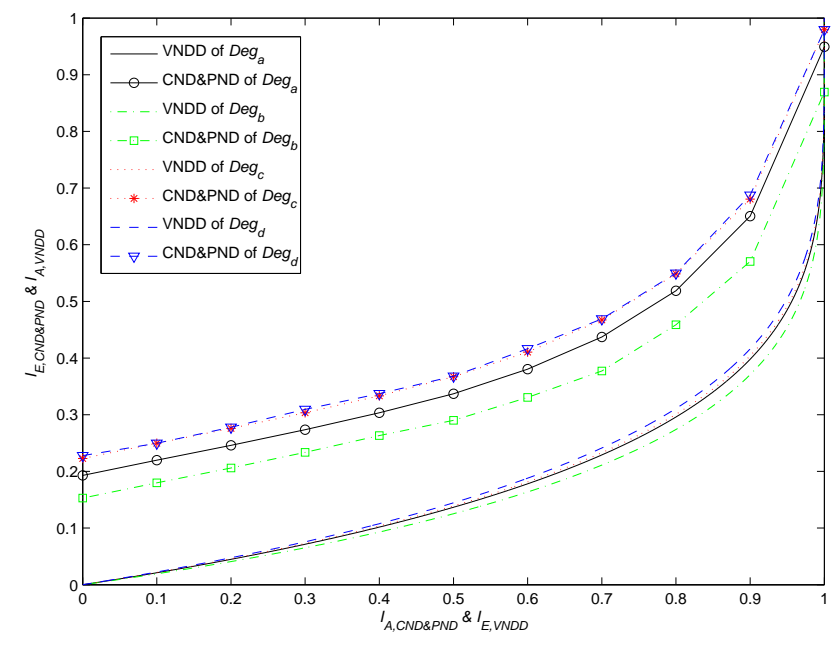

Fig. 7: EXIT chart for different degree distributions

\section{B. Short Cycle}

A cycle in a graph refers to a finite set of connected edges, the edge starts and ends at the same node, and it satisfies the condition that no node (except the initial and final node) appears more than once. Girth of a graph is the length of the shortest cycle. Apparently, girth should be equal to or greater than 4, and length-4 cycles manifest themselves in the corresponding matrix as four 1's that lie on the corners of a sub-matrix. Short cycles, especially length-4 cycles, degrade 
the performance of $\mathrm{MPA}^{4}$ [25]. Usually, short cycles are only considered and avoided in single graphs such as LDPC codes and LDS-OFDM. Nevertheless, in the joint sparse graph, length-4 cycles are easy to be regenerated without careful design. The results presented in Fig. 7 do not consider the impact of cycles, therefore, their girths equal to 4 .

Fig. 8 shows EXIT charts for different girth of the joint sparse graph, over ITU Pedestrian Channel B at $E_{b} / N_{0}=13$ $\mathrm{dB}$. It should be emphasized that the restriction of the girth is applied to the joint graph rather than any single graph. We choose the optimal $D e g_{d}$ to be the degree distribution. In the case when the girth equals to 6 , it is not difficult to remove length- 4 cycles by computer search. When the girth equals to 8 , the computer search is time-consuming to eliminate length- 4 and length- 6 cycles, and some degrees need to be modified marginally (labelled by $D e g_{d}^{\prime}$ ): $D_{C N D}(x)$ is $0.027 x^{4}+0.946 x^{5}+0.027 x^{6}, D_{P N D}(x)$ is $0.83 x^{3}+0.17 x^{4}$ and $D_{V N D D}(x)$ is $0.915 x^{4}+0.085 x^{5}$. It can be seen from this figure that curves of matrices with girth of 4 and 6 are very close to each other, whereas matrix with girth of 8 outperforms others. This is due to the same degree distributions adopted by matrices with girth of 4 and 6 , while the degree distribution of matrix with girth of 8 is slightly different. Although the EXIT chart only depends on the degree distribution, in order to improve system performance, we suggest to remove cycles of length of 4 and 6 when constructing a joint sparse graph, as short cycles may lead to failure of message convergence or oscillation between multiple states over repeat iterations. Ideally, in a cycle-free graph, the belief will converge to the exact a posteriori probability after a finite number of iterations. Nevertheless, cycles cannot be avoided, and the propagated information may lead to inaccurate a posteriori probability. Therefore, degree distribution and short cycle both affect the system performance.

\section{Vi. Performance Evaluation}

In this section, JSG-OFDM is simulated and compared with other well-known systems.

\section{A. Evaluation Configuration}

The performance of JSG-OFDM are evaluated and compared with existing well-known multiple access systems such as GO-MC-CDMA, LDS-OFDM and turbo structured LDSOFDM. The simulations are conducted over multipath fading channel, and the system parameters are listed in TABLE II. For fair comparisons, a half rate quasi-cyclic LDPC code with very low error floor and capacity approaching performance, is adopted by all the systems [26]. For GO-MC-CDMA, Welch-bound-equality (WBE) for loads of $200 \%$ and $300 \%$ is used, and the spreading codes are constructed by algorithms developed in [27]. The number of sub-carriers per group

\footnotetext{
${ }^{4}$ On the acyclic (without cycle) graph, the estimated marginal distribution actually converges to the true value in a finite number of iterations. Nevertheless, graph usually contains cycles. It is known that the graphs containing length- 4 cycles will converge in most cases, but the probabilities obtained might be incorrect. These graphs may fail to converge, or oscillate between multiple states over repeated iterations.
}

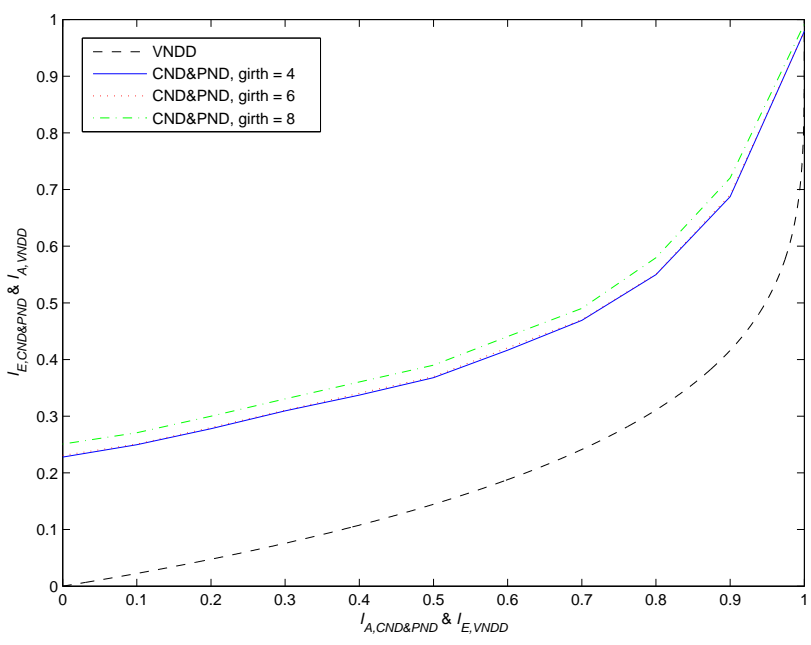

Fig. 8: EXIT chart for different schemes

is set to 4 and maximum likelihood detection is employed per group in GO-MC-CDMA. For LDS-OFDM, the LDS is optimized by EXIT chart, and an iterative detector is used [16]. For turbo structured LDS-OFDM, there are six outerinner turbo iterations between the detector and the decoder, and the turbo structure is optimized by EXIT chart in [17]. For JSG-OFDM, based on the analysis of Section V, three scenarios of the joint sparse graph that have different degree distribution and girth are given in TABLE IV. The maximum iterations are limited to six for LDS-OFDM and JSG-OFDM. Moreover, in the case when only one user is active in the uplink transmission, the theoretical single user bound can be obtained by the matched filter which optimally combines the transmitted symbol from all the paths, similar to a maximum ratio combiner, to maximize the SNR, and thereby minimizes error probability. All the investigated systems are compared to the optimal single user bound.

\section{TABLE IV: JSG-OFDM scenarios}

\begin{tabular}{|c|c|c|}
\hline Scenario & Degree distribution & Girth \\
\hline scenario -1 & Deg $_{a}$ & 6 \\
\hline scenario -2 & Deg $_{b}$ & 4 \\
\hline scenario -3 & Deg $_{d}^{\prime}$ & 8 \\
\hline
\end{tabular}

\section{B. BER Comparison}

Fig. 9 shows BER results for systems with a load of $200 \%$ over ITU Pedestrian Channel B. As we can see from this figure, performance of LDS-OFDM (Type-B receiver) is inferior to that of GO-MC-CDMA and turbo structured LDS-OFDM. Meanwhile, JSG-OFDM outperforms all the other systems. Due to the inherent advantage of the joint sparse graph, JSG-OFDM (Type-A receiver) achieves better performance than turbo structured LDS-OFDM (Type-C receiver). With the optimized degree distribution $\left(D e g_{d}^{\prime}\right)$ and cycle structure (girth of 8), JSG-OFDM scenario-3 achieves the best performance. Its performance improvements at BER of $10^{-5}$ are: $0.6 \mathrm{~dB}$ 
over JSG-OFDM scenario - $1,1.1 \mathrm{~dB}$ over JSG-OFDM scenario $-2,1.5 \mathrm{~dB}$ over turbo structured LDS-OFDM, $1.6 \mathrm{~dB}$ over GO-MC-CDMA and $1.8 \mathrm{~dB}$ over LDS-OFDM, respectively. Note that even if JSG-OFDM scenario - 3 is adopted, there is still a gap to the optimal single user bound. Moreover, for JSG-OFDM scenario - 3, physical layer framing is adopted and tested, i.e., 10 OFDM symbols constitute a frame. As its curve outperforms that of JSGOFDM scenario - 3, the joint sparse graph can be extended to fit the data length in practical systems.

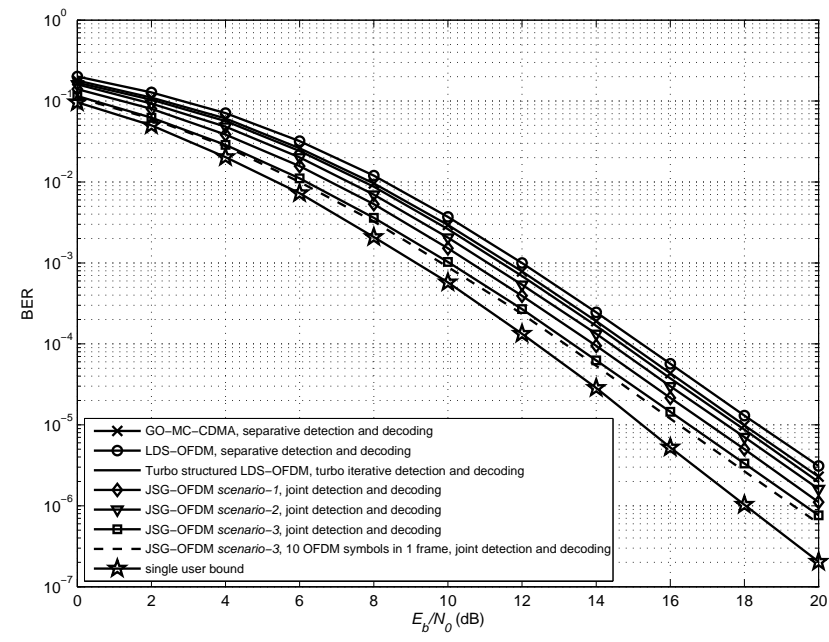

Fig. 9: Performance of $200 \%$ loaded systems over ITU Pedestrian Channel B

In addition, the performance for systems with a load of $300 \%$ over ITU Pedestrian Channel B is also evaluated and shown in Fig. 10. It can be seen that the BER results of $300 \%$ loading is inferior to that of $200 \%$ loading. For a load of $300 \%$, JSG-OFDM still outperforms other systems, and the scenario -3 of JSG-OFDM achieves the best performance. Compared with LDS-OFDM, GO-MC-CDMA and turbo structured LDS-OFDM, the JSG-OFDM scenario - 3 can obtain about $1.5-1.8 \mathrm{~dB}$ gain in the medium to high SNR region. Apparently, as the system loading increases, the gap to the optimal single user bound becomes wider.

To reveal the theoretical threshold of the joint sparse graph, we analyse its maximum achievable throughput by EXIT charts. It was proved in [23] that the capacity of an iterative system is equal to the area under the EXIT curve of the inner code, provided that the bit stream input to the receiver has independently distributed bits and the MAP algorithm is used for detection or decoding. For the joint sparse graph, assuming that the area under the EXIT curve of the CND\&PND is represented by $\mathcal{A}$, then the maximum achievable throughput at a particular $E_{b} / N_{0}$ value is given by $\mathcal{A}\left(E_{b} / N_{0}\right)$. In other words, if $\mathcal{A}$ is calculated for different $E_{b} / N_{0}$ values, the threshold of the joint sparse graph can be evaluated. Fig. 11 quantifies the maximum effective throughput of the joint sparse graph over ITU Pedestrian Channel B, where the SNR represent $E_{s} / N_{0}$, and the horizontal dotted line represents the throughput of the scheme considered. More explicitly,

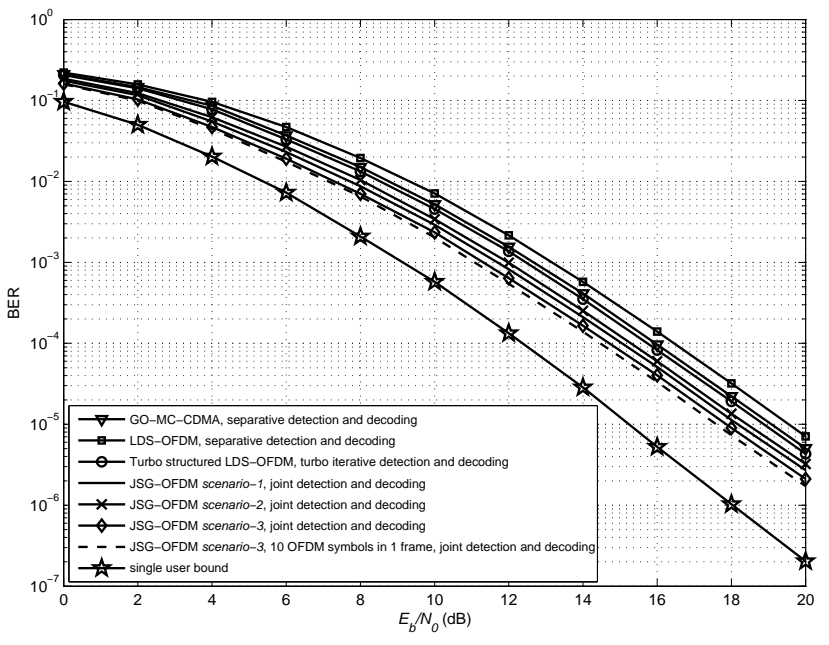

Fig. 10: Performance of $300 \%$ loaded systems over ITU Pedestrian Channel B

$0.5 \mathrm{bit} / \mathrm{symbol}$ is the effective throughput of half rate coded and BPSK modulated system. The circle and the rectangle are respectively located at the SNR required for $200 \%$ and $300 \%$ loaded JSG-OFDM scenario-3 to achieve an identical throughput at a target BER of $10^{-5}$. The SNR values shown next to the circle and the rectangle indicate the distance to the capacity. As can be seen that the $200 \%$ loaded JSG-OFDM scenario -3 is capable of operating within $1.1 \mathrm{~dB}$ from the capacity curve. When the system loading is increased to $300 \%$, the gap becomes $2.5 \mathrm{~dB}$. Hence, as expected, the lower the system loading employed, the closer the joint sparse graph operates to capacity.

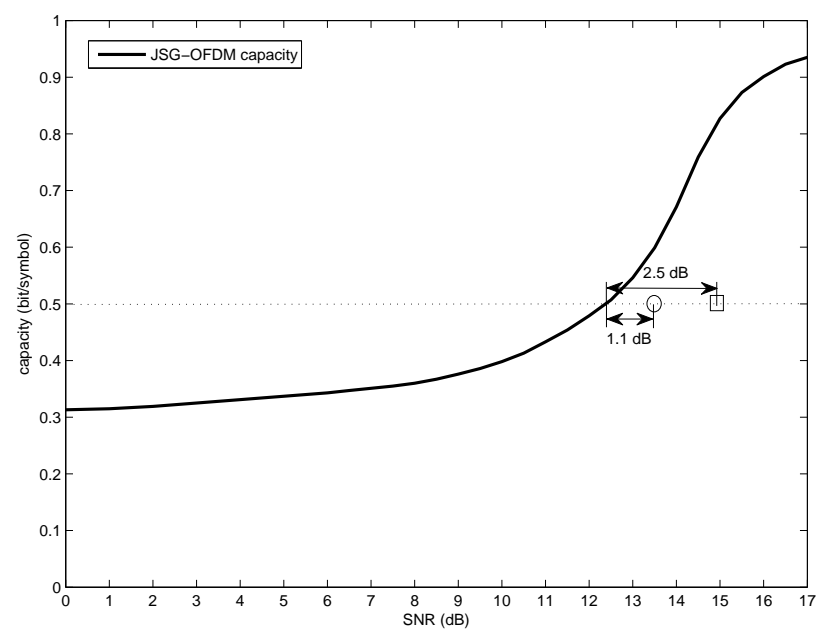

Fig. 11: Maximum effective throughput of JSG-OFDM over ITU Pedestrian Channel B

\section{Convergence Behavior}

Convergence behavior of JSG-OFDM is evaluated. Fig. 12 depicts the performance at different iterations of $200 \%$ 
loaded JSG-OFDM scenario - 1. As expected, over AWGN channel at $E_{b} / N_{0}=9 \mathrm{~dB}$, the BER stops falling down after 5 iterations, which is accurately predicted by the JMUDD trajectory presented in Fig. 4. Moreover, over ITU Pedestrian Channel B at $E_{b} / N_{0}=13 \mathrm{~dB}$, the receiver needs 4 iterations to reach the message convergence, which concurs with the JMUDD trajectory prediction plotted in Fig. 5. Therefore, EXIT chart analysis and convergence behavior of the joint sparse graph are verified by BER simulations.

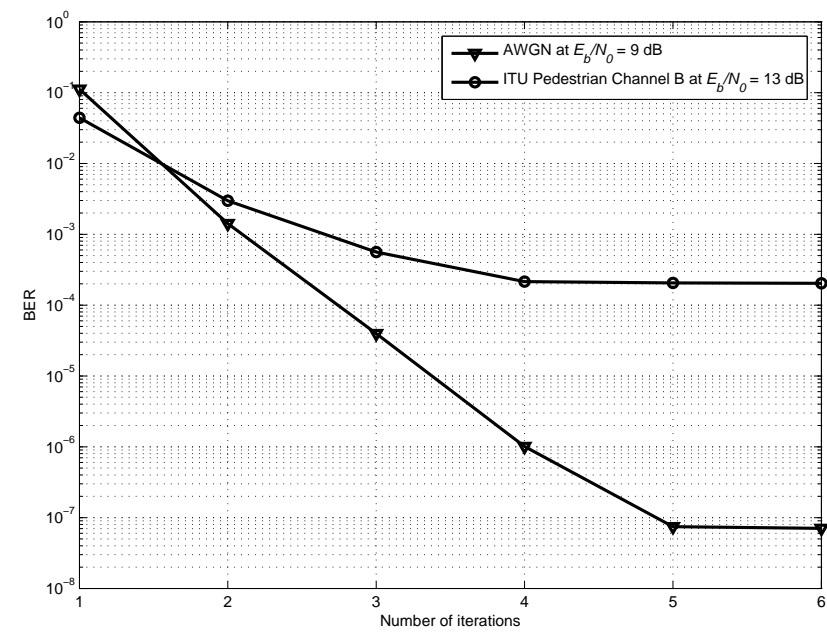

Fig. 12: Performance at different iterations for JSG-OFDM

\section{Performance of different users}

To gain more insight to the joint sparse graph, we present another result showing the performance of individual users. Fig. 13 illustrates the performance of the worst user and the best user in 200\% loaded JSG-OFDM scenario - 3 over ITU Pedestrian Channel B. It shows that some users have better performance than the others. To be more precise, we can see that at low SNR region the performance gap is not as obvious as in the high SNR region. This phenomenon can be explained by the dominating effect of noise at low SNRs.

\section{E. Near-far Effect}

The near-far problem is a condition in which a receiver captures a strong signal and thereby makes it impossible for the receiver to detect a weaker signal. The joint sparse graph does not give equal multiuser efficiency, and it does not result in the same performance for all the users as indicated by Fig. 13, so it is necessary to investigate the near-far effect of the JSG-OFDM. Fig. 14 shows the performance of near-far resistance for JSG-OFDM scenario -3 with different loads over ITU Pedestrian Channel B. The simulation is carried out for the case when $E_{b} / N_{0}=16 \mathrm{~dB}$ for the first user, and $E_{b} / N_{0}$ of other users is different. The BER of the first user is plotted against $\Delta E_{b} / N_{0}$ which represents the difference in $E_{b} / N_{0}$ between the user of interest and the other users. More explicitly, when the first user's SNR $E_{b} / N_{0}=16 \mathrm{~dB}$, the other users' SNR equals to $\Delta E_{b} / N_{0}$ plus $16 \mathrm{~dB}$. It can be seen that

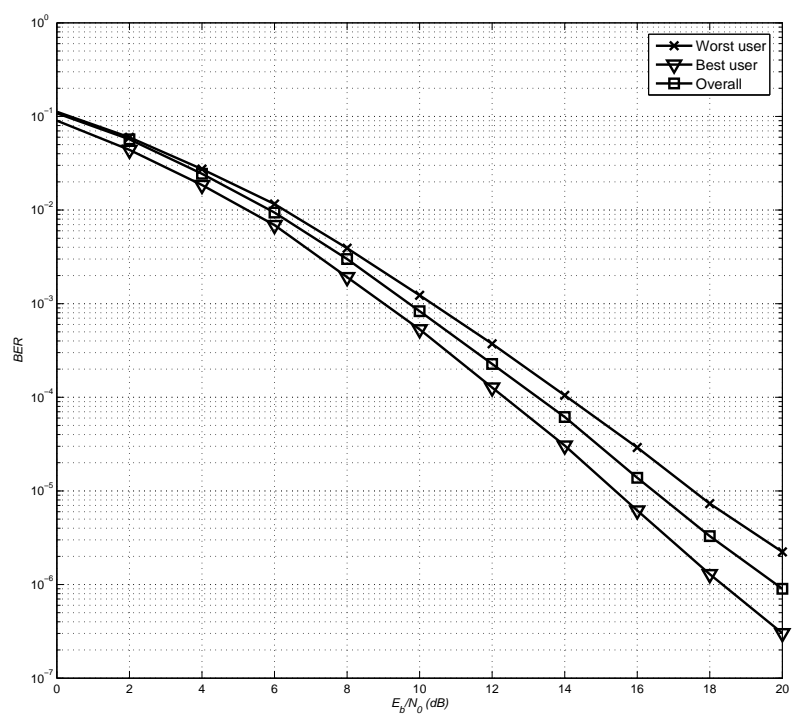

Fig. 13: Performance of different users in JSG-OFDM

unequal received power has a minor effect on the performance of user of interest under different loadings. It is due to the iterative processing, or in other words the near-far problem can be alleviated by the low density graph and the effective MPA. We can conclude that JSG-OFDM is robust against unequal received powers. To achieve better near-far resistances, the system can resort to the optimum power control mechanisms [28] [29].

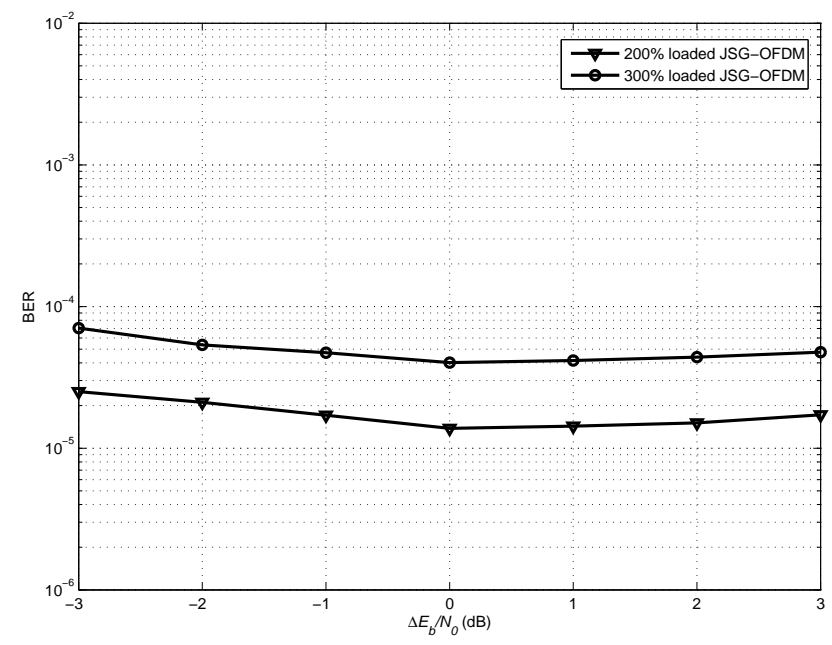

Fig. 14: Near-far effect of JSG-OFDM

\section{F. Multipath Diversity}

To test the multipath diversity of the joint sparse graph, we simulate the JSG-OFDM scenario-3 over different multipath channel model, i.e., ITU Pedestrian Channel A whose channel taps equal to four, and the ITU Pedestrian Channel B whose channel taps equal to six. Fig. 15 shows the comparison 
results. For JSG-OFDM scenario - 3, we can see that the performance of ITU Pedestrian Channel A is inferior to that of ITU Pedestrian Channel B, indicating that the multipath diversity is exploited by the JSG-OFDM system. However, to maximize the multipath diversity, other techniques such as linear constellation precoding [3] and multiple antennas [30], have to be applied to the JSG-OFDM if necessary.

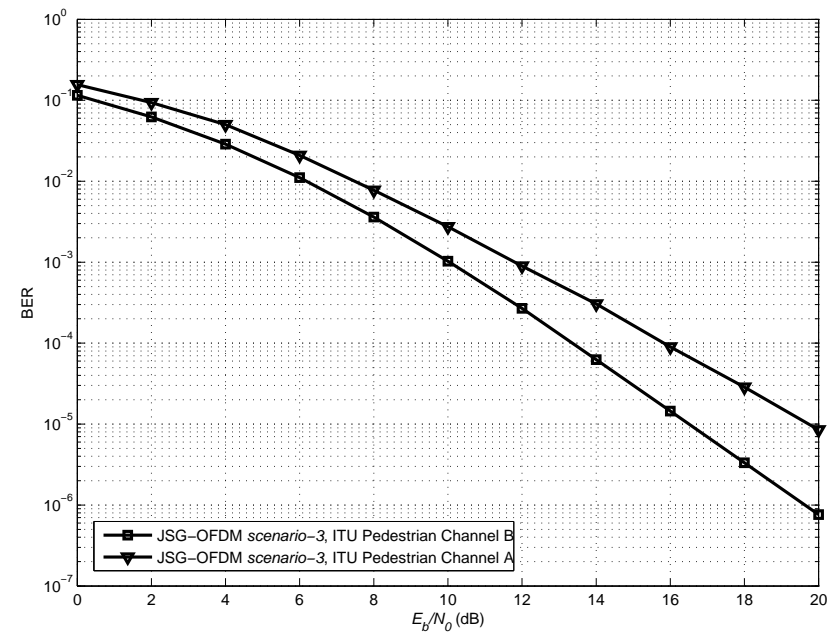

Fig. 15: Performance of JSG-OFDM over different multipath channels

\section{G. Detection Complexity Comparison}

As the same LDPC code is applied to all the investigated systems and the decoding complexity is therefore the same, we focus on the comparisons of detection complexity. Let $\mathbb{X}$ be the constellation alphabet for the transmitted symbol, which is related to the modulation order. In GO-MC-CDMA, the detector complexity increases exponentially with the number of data symbols in each group, which results in a complexity order of $\mathcal{O}\left(|\mathbb{X}|^{8}\right)$. In LDS-OFDM, the detector complexity increases exponentially with the number of symbols per subcarrier, its complexity order is $\mathcal{O}\left(|\mathbb{X}|^{6}\right)$. In turbo structured LDS-OFDM, the detector complexity is several times higher than that of LDS-OFDM. Apparaently, the complexity of the LDS-OFDM detector is less than that of GO-MC-CDMA and turbo structured LDS-OFDM. Thus, we compare the detection complexity between JSG-OFDM and LDS-OFDM. It is noteworthy that due to the similar message passing algorithm, the detection complexity order of the JSG-OFDM is $\mathcal{O}\left(|\mathbb{X}|^{6}\right)$ which is the same as that of LDS-OFDM. However, as the convergence behavior and the intersection point of the EXIT chart are different between LDS-OFDM and JSGOFDM, the detection complexity is slightly different, which will be discussed in the sequel.

We express the complexity of detection in terms of equivalent additions. The basic operations performed by MPA include addition (ADD), subtraction (SUB), multiplication by \pm 1 (MUL), division by 2 (DIV), comparison (CP) and $\max (x, y)(\mathrm{MAX})$. The ADD, SUB, MUL, DIV and CP operations correspond to one equivalent addition, and the MAX operation corresponds to two equivalent additions, since it first uses a CP operation to compare the two input values and then stores the result in a register [31]. TABLE V summarizes the computational requirements for a load of $200 \%$ over ITU Pedestrian Channel B. On one hand, for LDS-OFDM and JSG-OFDM (including scenario -1 and scenario -3 ), the equivalent addition operations drop dramatically when $E_{b} / N_{0}$ increases, which means channel conditions affect the number of iterations significantly. This is due to the property of the message passing algorithm, more explicitly, as shown in the JMUDD, the iteration can be terminated promptly if the syndrom equals to zero. Therefore, the number of addition operations is a function of $E_{b} / N_{0}$. On the other hand, it is more costly to perform multiuser detection in JSG-OFDM scenario -1 than in LDS-OFDM. However, by carefully design of the joint sparse graph, the detection complexity of JSG-OFDM scenario -3 is less than that of JSG-OFDM scenario -1 and LDS-OFDM. This is related to the optimization of degree distributions which reduces the density of the graph.

TABLE V: Equivalent number of addition operations for detection

\begin{tabular}{|c|c|c|c|c|c|}
\hline System & $4 \mathrm{~dB}$ & $8 \mathrm{~dB}$ & $12 \mathrm{~dB}$ & $16 \mathrm{~dB}$ & $20 \mathrm{~dB}$ \\
\hline LDS-OFDM & 249019 & 179565 & 112956 & 85971 & 63502 \\
\hline JSG-OFDM scenario -1 & 249465 & 180072 & 113753 & 86522 & 64123 \\
\hline JSG-OFDM scenario -3 & 244013 & 176136 & 111266 & 84631 & 62721 \\
\hline
\end{tabular}

\section{CONCLUSION}

In this paper, a JSG-OFDM system has been proposed and analysed. Unlike any previous single graph, the JSG-OFDM is based on a joint sparse graph which combines multiple access (LDS-OFDM) and FEC coding (LDPC codes). The system framework of JSG-OFDM is designed, and the low complexity JMUDD, which applies MPA on the joint sparse graph, is presented. JSG-OFDM is a novel multiple access system that jointly performs detection and decoding on one entire sparse graph. In addition, the iterative structure of JSG-OFDM receiver is illustrated. Its convergence behavior is analysed by EXIT charts and indicated by the JMUDD trajectories over different channels. Furthermore, degree distribution and short cycle of the joint sparse graph are investigated using EXIT chart. It is shown that these two parameters affect the performance of JUMDD significantly. According to the design guidelines of the joint sparse graph, three scenarios of JSGOFDM are presented, and similar well-known multiple access systems are compared. The simulation results show that the JSG-OFDM scenario - 3 (degree distribution of $D e g_{d}^{\prime}$ and girth of 8) achieves the best performance. Its performance improvement is mainly due to the offline optimization of the joint sparse graph to exploit frequency domain diversity in addition to avoiding strong interference to corrupt all the sub-carriers. In general, JSG-OFDM can improve system performance and brings about $1.5-1.8 \mathrm{~dB}$ gain. The joint sparse graph has to be carefully designed for specific applications, and needs 
to be considered in MIMO transmissions, which needs further investigations.

\section{ACKNOWLEDGMENT}

The authors would like to thank Jing Lei, Jibo Wei and Dandan Liang for their constructive and insightful comments. The authors would also like to thank the 5th Generation (5G) Mobile Communications project by $5 \mathrm{G}$ innovation centre (5GIC) in the University of Surrey, the United Kingdom Engineering and Physical Science Research Council (EPSRC) under grant number EP/J017655/1 and the National Natural Science Foundation of China (NSFC) under grant number 61372098 for their financial support.

\section{REFERENCES}

[1] 3GPP, "Physical layer aspects for evolved Universal Terrestrial Radio Access (UTRA)," 3GPP Std. TR 25.814 v.7.0.0, Tech. Rep., 2006.

[2] IEEE, "Standard for local and metropolitan area networks. Part 16: Air interface for broadband wireless access systems," Tech. Rep., May 2009.

[3] Z. Liu, Y. Xin, and G. B. Giannakis, "Linear constellation precoding for OFDM with maximum multipath diversity and coding gains," IEEE Transactions on Communications, vol. 51, no. 3, pp. 416-427, March 2003.

[4] D. N. C. Tse and S. V. Hanly, "Linear multiuser receivers: effective interference, effective bandwidth and user capacity," IEEE Transactions on Information Theory, vol. 45, no. 2, pp. 641-657, August 1999.

[5] K. Zhang, Y. L. Guan, and Q. Shi, "Complexity reduction for MCCDMA with MMSEC," IEEE Transactions on Vehicular Technology, vol. 57, no. 3, pp. 1989-1993, May 2008.

[6] A. L. Sacramento and W. Hamouda, "Multiuser decorrelator detectors in MIMO CDMA systems over Nakagami fading channels," IEEE Transactions on Wireless Communications, vol. 8, no. 4, pp. 1944-1952, May 2009.

[7] R. Ming, M. C. Reed, and S. Zhenning, "Successive multiuser detection and interference cancelation for contention based OFDMA ranging channel," IEEE Transactions on Wireless Communications, vol. 9, no. 2, pp. 481-487, February 2010.

[8] B. A. Al-fuhaidi, H. E. A. Hassan, M. M. Salah, and S. S. Alagooz, "Parallel interference cancellation with different linear equalisation and rake receiver for the downlink MC-CDMA systems," IET Communications, vol. 6, no. 15, pp. 2351-2360, March 2012.

[9] P. H. Tan and L. K. Rasmussen, "Asymptotically optimal nonlinear MMSE multiuser detection based on multivariate Gaussian approximation," IEEE Transactions on Communications, vol. 54, no. 8, pp. 14271438, August 2006.

[10] H.-Y. Lu, "Iterative multiuser detectors for spatial-frequency-timedomain spread multi-carrier DS-CDMA systems," IEEE Transactions on Vehicular Technology, vol. 60, no. 4, pp. 1640-1650, May 2006.

[11] M. Mukherjee and P. Kumar, "Overloaded WH-spread CI MC-CDMA with iterative interference cancellation," IEEE Communications Letters, vol. 15, no. 12, pp. 1391-1393, December 2011.

[12] C. Xiaodong, Z. Shengli, and G. B. Giannakis, "Group-orthogonal multicarrier CDMA," IEEE Transactions on Communications, vol. 52, no. 1, pp. 90-99, January 2004.

[13] F. Riera-Palou, G. Femenias, and J. Ramis, "On the design of uplink and downlink group-orthogonal multicarrier wireless systems," IEEE Transactions on Communications, vol. 56, no. 10, pp. 1656-1655, October 2008

[14] A. Montanari and D. Tse, "Analysis of belief propagation for non-linear problems: The example of CDMA (or: How to prove tanakas formula)," in IEEE Information Theory Workshop, March 2006.

[15] R. Hoshyar, F. P. Wathan, and R. Tafazolli, "Novel low-density signature for synchronous CDMA systems over AWGN channel," IEEE Transactions on Signal Processing, vol. 56, no. 4, pp. 1616-1626, April 2008.

[16] R. Hoshyar, R. Razavi, and M. Al-Imari, "LDS-OFDM an efficient multiple access technique," in IEEE 71st Vehicular Technology Conference, May 2010.

[17] R. Razavi, M. Al-Imari, M. A. Imran, R. Hoshyar, and D. Chen, "On receiver design for uplink low density signature OFDM (LDS-OFDM)," IEEE Transactions on Communications, vol. 60, no. 11, pp. 3499-3508, November 2012.
[18] R. Koetter, A. C. Singer, and M. Tuchler, "Turbo equalization," IEEE Signal Processing Magazine, vol. 21, no. 1, pp. 67-80, January 2004.

[19] R. G. Gallager, "Low-density parity-check codes," IRE Transactions on Information Theory, vol. 8, no. 1, pp. 21-28, January 1962.

[20] E. Sharon, A. Ashikhmin, and S. Litsyn, "Analysis of low-density parity-check codes based on EXIT functions," IEEE Transactions on Communications, vol. 54, no. 8, pp. 1407-1414, August 2006.

[21] S. ten Brink, "Convergence behavior of iteratively decoded parallel concatenated codes," IEEE Transactions on Communications, vol. 49, no. 10 , pp. 1727-1737, October 2001.

[22] R. Diestel, "Graph theory," Springer-Verlag Heidelberg, Tech. Rep., 2005.

[23] L. Hanzo, T. Liew, B. Yeap, R. Tee, and S. Ng, "Turbo coding, turbo equalisation and space-time coding: EXIT-chart-aided near-capacity designs for wireless channels, decond edition," John Wiley \& Sons IEEE Press, Tech. Rep., 2011.

[24] M. El-Hajjar and L. Hanzo, "EXIT charts for system design and analysis," IEEE Communications Surveys \& Tutorials, vol. 16, no. 1, pp. 127-153, February 2014.

[25] H. Aymeersch, F. Penna, and V. Savic, "Uniformly reweighted belief propagation for estimation and detection in wireless networks," IEEE Transactions on Wireless Communications, vol. 11, no. 4, pp. 15871595, April 2012.

[26] C. Jinghu, R. M. Tanner, Z. Juntan, and M. P. C. Fossorier, "Construction of irregular LDPC codes by quasi-cyclic extension," IEEE Transactions on Information Theory, vol. 53, no. 4, pp. 1479-1483, April 2007.

[27] S. Ulukus and R. D. Yates, "Iterative construction of optimum signature sequence sets in synchronous CDMA systems," IEEE Transactions on Information Theory, vol. 47, no. 5, pp. 1989-1998, July 2001.

[28] W. Zhuwei, Y. Dacheng, and L. Milstein, "Multi-user resource allocation for a distributed multi-carrier DS-CDMA network," IEEE Transactions on Communications, vol. 60, no. 1, pp. 143-152, January 2012.

[29] C. Chih-Wen and K. Chien-Cheng, "A novel interference-avoidance code reassignment for downlink two-dimensional-spread MC-DS-CDMA systems with power control," IEEE Transactions on Vehicular Technology, vol. 59, no. 6, pp. 3104-3108, July 2010.

[30] G. V. Rangaraj, D. Jalihal, and K. Giridhar, "Exploiting multipath diversity in multiple antenna OFDM systems with spatially correlated channels," IEEE Transactions on Vehicular Technology, vol. 54, no. 4, pp. 1372-1378, July 2005.

[31] I. A. Chatzigeorgiou, M. R. D. Rodrigues, I. J. Wassell, and R. A. Carrasco, "Comparison of convolutional and turbo coding for broadband FWA systems," IEEE Transactions on Broadcasting, vol. 53, no. 2, pp. 494-503, June 2007. 